\title{
Transplantation of Mesenchymal Stem Cells for Prevention of Acute Myocardial Infarction Induced Heart Failure: Study Protocol of a Phase III Randomized Clinical Trial (Prevent-TAHA8)
}

\section{Armin Attar ( $\square$ attar_armin@yahoo.com )}

Shiraz University of Medical Sciences https://orcid.org/0000-0002-4133-4870

Ahmad Monabati

Shiraz University of Medical Sciences

Mohammad Montaseri

Shiraz University of Medical Sciences

Massoud Vosough

Royan Institute for Stem Cell Biology and Technology

Seyed Ali Hosseini

Shiraz University of Medical Sciences

Javad Kojouri

Shiraz University of Medical Sciences

Alireza Abdi-Ardekani

Shiraz University of Medical Sciences

Peyman Izadpanah

Shiraz University of Medical Sciences

Negar Azarpira

Shiraz University of Medical Sciences

Gholamreza Pouladfar

Shiraz University of Medical Sciences

Mani Ramzi

Shiraz University of Medical Sciences

\section{Research Article}

Keywords: regenerative medicine, cell therapy, myocardial infarction, mesenchymal stem cells, intracoronary injection, acute myocardial infarction

Posted Date: January 7th, 2022 
DOI: https://doi.org/10.21203/rs.3.rs-1008866/v1

License: (c) (1) This work is licensed under a Creative Commons Attribution 4.0 International License. Read Full License 


\section{Abstract}

\section{Background}

Results from recent clinical trials on bone marrow mononuclear cell (BM-MNC) transplantation show that this intervention can help reduce the incidence of heart failure (HF) after acute myocardial infarction (AMI). However, no study has evaluated the effect of the transplantation of mesenchymal stem cells (MSCs) on a clinical endpoint such as HF.

\section{Methods}

This single-blinded, randomized, multicenter trial aims to establish whether the intracoronary infusion of umbilical cord-derived Wharton's jelly MSCs (WJ-MSCs) helps prevent HF development after AMI. The study will enroll 360 patients 3 to 7 days following AMI. Only patients aged below 65 years with impaired LV function (LVEF < 40\%) will be included. They will be randomized (2:1 ratio) to either receive standard care or a single intracoronary infusion of $10^{7}$ WJ-MSCs. The primary outcome of this study is the assessment of HF development during long-term follow-up (3 years).

\section{Discussion}

Data will be collected until Nov 2024. Thereafter, the analysis will be conducted. Results are expected to be ready by Dec 2024. We will prepare and submit the related manuscript in accordance with the SPIRIT guidelines. This study will help determine whether or not the infusion of intracoronary WJ-MSCs in patients with AMI will reduce the incidence of AMI-induced HF.

Trial Registration

ClinicalTrials.gov, NCT05043610, Registered 14 September 2021 - Retrospectively registered, https://clinicaltrials.gov/ct2/show/NCT05043610

\section{Introduction}

Myocardial infarction (MI) represents a leading cause of mortality worldwide (1). With a reduction in the rate of mortality due to Mls in recent decades, the incidence of heart failure (HF) has been on the rise (2). This incidence ranges between 14 and $36 \%$ among those hospitalized due to an acute MI (AMI) (3). HF exerts a considerable effect on healthcare systems in America, accounting for 6 million cases, 300,000 deaths, and roughly 40 billion USD worth of costs every year (4).

Despite the therapeutic efforts (5), post-MI HF still leads to a high rate of morbidities and mortalities (6, 7). Although we have been successful in prolonging the life of HF patients and relieving symptoms, we are yet to regenerate the infarcted cardiac tissues. Hence, a gap exists in the literature as restoring the standard histological architecture of the heart should theoretically lead to improved outcomes for patients with Ml-induced HF (8). This may be possible using stem cell-based therapies (9). 


\section{Cell-based therapy in cardiovascular disease}

Toward the close of the $20^{\text {th }}$ century, scientists signaled a new era in cardiovascular disease treatment through preclinical investigations in which skeletal myoblasts (10) and fetal cardiomyocytes (11) were transplanted into ischemic myocardium. Afterward, the intracardiac implantation of bone marrow (BM) cells was assessed in murine MI models $(12,13)$. Human studies commenced following the turn of the century, with skeletal myoblasts being used in HF patients in 2001 (14) and BM cells being used for AMI patients in 2002 (15). From then on, numerous investigations have aimed to amend the cardiovascular damage caused by diseases like $\mathrm{MI}$ and cardiomyopathy through the use of different cell-based therapies.

\section{Mesenchymal stem cells (MSCs)}

The BM, heart, Wharton's jelly, and adipose tissue are among the prime sources of MSCs (16)(17)(18). MSCs offer ease of isolation, ex vivo growth, in vitro proliferation, and immune-privileged properties, which is why their use is in clinical trials is expanding rapidly (19). According to the POSEIDON clinical trial on MSC transplantation, allogeneic MSCs are safe and as effective as autologous MSCs (20). Notably, the TAC-HFT trial revealed the two-fold effectiveness of MSCs relative to BM-derived mononuclear cells (BM-MNCs) (21). Accordingly, MSCs appear to be an excellent candidate for cardiac regeneration trials.

\section{Cell-based therapy in acute myocardial infarction (AMI)}

To date, BM-MNCs have been used in the majority of research on cell-based therapy following AMI. The TIME trials established that the optimal time for cell implantation following AMI is within 3-7 days (22, 23). Fisher et a., in a meta-analysis, proved that BM-MNCs augment the left ventricular ejection fraction (LVEF) following AMI by roughly $2.72 \%$, yielding benefits both in terms of survival and function in AMI patients younger than 55 years of age with LVEF $<37 \%(24)$.

Trials involving the use of MSCs in patients following AMI have shown promising yet controversial results. Gao and coworkers conducted the largest clinical trial in this regard with 116 patients, demonstrating that umbilical cord-derived Wharton's jelly MSCs (WJ-MSCs) led to an almost five percent improvement in the LVEF (25). This figure was held slightly lower at $3.84 \%$ in a related meta-analysis (26). These are in agreement with the findings of the TAC-HFT trial, which indicated the roughly two-fold effectiveness of MSCs relative to BM-MNCs (21).

\section{BAMI trial}

For over two decades, autologous cell-based treatments have been assessed in managing cardiovascular diseases through preclinical and clinical studies. However, phase III trials have been infrequent. 
Furthermore, the phase II trials have involved different methodologies in terms of the type of stem cells and the method and timing of delivery.

The BAMI trial was the first phase III trial conducted to clarify whether or not post-MI intracoronary transplantation of BM-MNCs would reduce all-cause mortality. Although the trial was designed to involve 3000 patients, it was stopped prematurely due to futility after the enrollment of 375 patients. Among them, 185 received BM-MNCs (intracoronary infusion) 2-8 days following primary percutaneous coronary intervention (PPCI), and the remaining 190 patients received optimal medical therapy as the control group. All-cause mortality after two years was 3.26\% [n=6; 95\% confidence interval (Cl): 1.487.12\%] with BM-MNCs compared to $3.82 \%(n=7 ; 95 \% \mathrm{Cl}: 1.84-7.84 \%)$ with optimal medical therapy. The main reason behind such results was a significant reduction in post-AMI mortality. At the start of the project in 2011, the literature held that following an AMI, the mortality rate from all causes after two years would be approximately $12 \%$ among those with an LVEF $\leq 45 \%$ post-reperfusion therapy (3). However, the researchers noticed a $3.85 \%$ mortality rate while conducting the study, reflecting the evolution of primary angioplasty procedures in those years. Importantly, the investigators noticed that only five patients $(2.7 \%, 95 \% \mathrm{Cl}: 1.0-5.9 \%)$ who received BM-MNCs were hospitalized due to HF during the two years of follow-up compared with 15 patients $(8.1 \%, \mathrm{Cl}$ : $4.7-12.5 \%)$ who received optimal medical therapy (HR: $0.33,95 \% \mathrm{Cl}: 0.12-0.88)$, representing the sole clinical benefit observed. BAMI showed us that taking mortality as an endpoint for stem cell therapy trials is futile, and the best clinical endpoint to assess is $\mathrm{HF}$ incidence.

\section{Hypothesis generation}

Since the efficacy of MSCs is higher than BM-MNCs after AMI in the improvement of LVEF, it would be probable that these cells may have a better clinical effect as well. However, no study has evaluated the impact of the transplantation of MSCs on a clinical endpoint such as HF.

\section{Materials And Methods}

\section{Study design}

A randomized, multicenter, single-blinded phase III trial will be conducted to assess whether the intracoronary infusion of umbilical cord WJ-MSCs demonstrates a superior effect in reducing HF incidence following AMIs compared to standard treatment. The Ethics Committee of Shiraz University of Medical Sciences approved the study protocol (code: IR.SUMS.REC.1400.409). The trial is registered with https://clinicaltrial.gov under the code NCT05043610. This protocol was conceived following the Standard Protocol Items: Recommendations for Interventional Trials (SPIRIT) guidelines (Online supplement 1). Figure 1 depicts the SPIRIT flow diagram of the study.

\section{Sample size}


In line with the related literature, we made the following assumptions in determining the sample size required considering the aims of this phase III randomized clinical trial: $5 \%$ error, $80 \%$ power, a one-year $\mathrm{HF}$ incidence rate of $1.3-4 \%$, and division between two groups in a 1:1 ratio. Using the formula

$$
n=\frac{2 \bar{P}(1-\bar{P})\left(t_{\alpha, Y}+t_{\beta(1), V}\right)^{2}}{(\partial)^{2}}, 272
$$

people in each group were estimated for a follow-up period of two years and 108 people in each group for four years or 328 patients in 2:1 ratio (control/case) for 3 years. This sample size is only to compare the incidence rate based on the hypothesis test. If we intend to use the Cox regression model, ten people should be added for each independent variable.

\section{Study participants}

A total of 360 patients with a history of an anterior ST-elevation MI (STEMI) treated successfully with PPCI 3-7 days earlier will be enrolled. Patients must be below 65 years old and must have severely impaired function of the left ventricle (LV), represented by an LVEF of $<40 \%$. The participating hospitals will be the Al-Zahra Heart, Namazee, and Faghihi hospitals of Shiraz, Iran.

The inclusion criteria are as follows:

1. Age (years): 18 to 65

2. Either gender

3. First MI in the preceding 3 to 7 days

4. Post-AMI LVEF $<40 \%$

5. Negative pregnancy test (for women of reproductive age)

6. Written informed consent

The exclusion criteria are as follows:

1. A history of any prior cardiac conditions (valvular, ischemic, or congenital disorders)

2. Regional wall motion abnormalities outside the region of the infarction

3. LV dysfunction due to other etiologies like non-ischemic cardiomyopathy, anthracycline use, or ethanol abuse (> 6 oz./day regularly)

4. Poor echocardiography window

5. Active infection, malignancy, or autoimmune disease

\section{Randomization and blinding}

Randomization will be done via permuted block randomization through a web-based service. A block size of 4 will be considered. Two groups of with 2:1 proportion will be formed, where only one will receive an 
intracoronary infusion of WJ-MSCs besides the conventional therapy provided to both groups. Those who assess the study outcomes will remain unaware of the allocation (single-blind).

\section{Intervention}

This study will use cGMP-certified clinical-grade human WJ-MSCs (Cell Tech Pharmed Co. Ltd., Tehran, Iran). The cells will be transferred to the hospital on the same day as the infusion and are to be suspended in normal saline (0.9\%). Each batch will be analyzed and certificated by a qualified individual, ensuring that the cells comply with the product specifications. Standard operational protocols will be followed during shipment and handling.

In the intervention group, all 120 patients will receive a single intracoronary infusion of $10^{7}$ WJ-MSCs alongside the conventional treatment that will be provided to the same number of patients in the control group. Patients in the intervention group will be taken to the cardiac catheterization lab, where the infusion of $10^{7}$ WJ-MSCs will be done through the intracoronary route. A bolus heparin dose (weightbased) is to be administered to patients with an activated clotting time below 200 seconds.

For catheterization, we will insert a therapeutic 6 Fr guiding catheter into the left coronary artery. Following the infusion of nitroglycerin $(200 \mu \mathrm{g})$ through the catheter, we will assess the left anterior descending (LAD) artery and document the TIMI flow. A 0.014-inch soft-tipped guidewire wire will be inserted into the LAD at the distal edge of the stent. After passing through an over-the-wire balloon to the stented area, the balloon will be inflated until achieving occlusion. Following the removal of the guiding wire, we will connect an infusion syringe to the infusion catheter. WJ-MSCs will be infused at a rate of 2.5 $\mathrm{ml} / \mathrm{min}$. Low-pressure inflation (2-4 bar) will be performed to achieve occlusion with the balloon catheter, with complete coronary artery occlusion being ensured ahead of cell infusion through the use of dye. After the infusion of each third of the cells, we will pause the infusion, and TIMI coronary flow will be assessed with the contrast agent before the resumption of cell infusion. Once the cells are delivered across the three portions, we will place the coronary flow wire via the microinfusion catheter.

\section{Follow-up and endpoints}

Patients will receive daily visits from a cardiologist during hospitalization. The results of all physical examinations will be recorded, and patients will be monitored for early manifestations signaling arrhythmia, pulmonary embolism, or coronary artery injury. Blood tests will be done to measure fasting blood sugar, complete blood count, C-reactive protein, urea and electrolytes, liver function test, creatine kinase, and cardiac troponin T. An electrocardiogram (ECG) will also be obtained. Prior to the MSC infusion process, the cardiac evaluation will be completed using cardiac magnetic resonance imaging and echocardiography. The initial EF will be established according to the wall motion score and Simpson's rule. After the MSCs are delivered, once stable, a beta-blocker, angiotensin-converting enzyme (ACE) inhibitor, aldosterone antagonist, aspirin, ticagrelor, statin, and glyceryl trinitrate (spray or tablets) 
will be prescribed for the patient to use at home. A cardiac rehabilitation program will also be completed. Subsequent visits will be at ten days after discharge and then every three months, where an ECG and blood tests will be requested. Echocardiography will be done during the six-month follow-up and the final visit, facilitating the evaluation of LV systolic function.

Our primary endpoint to assess the efficacy of the intervention will be the incidence of HF. Secondary endpoints include the improvement in LV function (through calculation of LVEF) after six months and after three years alongside echocardiographic changes in the left ventricular mass, left ventricular enddiastolic volume, left ventricular end-systolic diameter, and global longitudinal strain (measured via automated formulas in standard views) indices.

\section{Adjudication of study measures}

Before statistical analysis, adjudication of all measurements will be done by an experienced cardiology department member excluded from the research group. The adjudicator will assess the quality of each measurement and will exclude those with inadequate quality from the analysis, where they will be regarded as missing. An independent, blinded safety committee will evaluate potential major adverse cardiac events (MACEs). Once the adjudication process is complete, the finalized database will be unblinded.

\section{Statistical analysis}

Data will be kept anonymous until analysis, which is to be performed by an independent statistician external to the research group. Treatment efficacy will be assessed according to the decrement in HF with the help of Cox regression analysis. We will consider the EF to have improved significantly if a minimum increment of $3 \%$ is achieved after six months. The analysis will follow the intention-to-treat approach. The baseline characteristics of the two study groups will also be compared. Continuous variables will be summarized using the mean and standard deviation, while frequencies and percentages will be given for categorical data. The $\mathrm{EF}$, as the primary outcome, will be compared between the study groups using the independent t-test and one-way analysis of variance (ANOVA). The therapeutic effect will be estimated with a $95 \% \mathrm{Cl}$. Two-sided P-values will be used. Safety will be compared between the two groups according to the occurrence of MACEs (death, recurrent AMI, ICD insertion, non-target vessel revascularization, etc.) and serious adverse events (SAEs). These events will be followed over time with Kaplan-Meier curves, which will allow us to understand their patterns. With the help of the Cox proportional hazards model, we will assess the statistical significance and 95\% $\mathrm{Cl}$.

\section{Adverse events}

Adverse events will be reported by the study's executive committee to an independent Data and Safety and Monitoring Board (DSMB). The DSMB will have the authority to stop the trial early if patient safety is 
compromised or if the primary research objective is met. All safety issues (unanticipated SAEs, mortality, intracoronary infusion complications, and severe arrhythmias, etc.) will be monitored by the DSMB, and the DSMB statistician will report the occurrence of safety issues in each study group quarterly. All deaths will be reported.

\section{Ethical considerations}

We discussed all ethical issues with the Institutional Review Board of Shiraz University of Medical Sciences, which ultimately approved the study protocol (IR.SUMS.REC.1400.409). Informed consent will be obtained once patients are clinically stable and sedatives or strong analgesics do not alter their consciousness. Importantly, the use of low balloon inflation pressure and divided (three-part) infusions will prevent complications related to intracoronary cell infusion. The principles of the Declaration of Helsinki will be upheld throughout this study.

\section{Results}

Currently, the primary focus of post-AMI treatment is to prevent remodeling and avert any further loss of myocytes (5). However, a revolution can potentially be achieved by regenerative medicine, aiming to restore cardiac function by inhibiting and even reversing the process of remodeling through the use of stem cells (25). Despite the fact that some investigations were not very promising in this regard (9), other studies have shown that stem cell therapy may be of value in certain populations.

Although a Cochrane meta-analysis revealed that the LVEF of young AMI patients does not increase following BM-MNC therapy, survival and functional benefits may be present (24). Importantly, research with MSCs has yielded more promising results, with the TAC-HFT trial indicating the roughly two-fold higher efficacy of MSCs relative to BM-MNCs (21). According to meta-analyses of the various clinical trials, MSCs can improve the LVEF by $3.84 \%$ (26), while BM-MNCs can achieve an inferior improvement of $2.72 \%(24)$.

Currently, scientists are yet to understand the exact mechanisms behind the therapeutic impact of stem cells, especially MSCs. However, the most commonly suggested mechanism is paracrine signaling, where the implanted stem cells alter the activity of the nearby cells in the heart via mediators like cytokines (27). Also, it is still unclear whether or not the mechanical improvements in LV function after MSC transplantation would be translated into a clinical benefit by reducing major cardiovascular events. Our trial provides essential insights into the field by including selected patients who develop reduced LVEFs after AMls.

Our trial by, enrolling 360 patients, would be the largest clinical trial ever conducted in the field of regenerative medicine in cardiology. We hope it would help clarify whether MSC transplantation is clinically useful or not. 


\section{Trial Status}

We began enrolling patients in Jan 2021 and expect to finish the recruitment process by Nov 2022. Data will be collected until Nov 2024. Thereafter, the analysis will be conducted. Results are expected to be ready by Dec 2024. We will prepare and submit the related manuscript in accordance with the SPIRIT guidelines. This study is registered with clinicaltrials.gov under the code NCT05043610. This protocol (version 1) was approved in Oct 2020.

\section{Abbreviations}

Acute myocardial infarction (AMI)

Bone marrow (BM)

Bone marrow-derived mononuclear cells (BM-MNCs)

Data and Safety and Monitoring Board (DSMB)

Ejection fraction (EF)

Heart failure (HF)

Left ventricular ejection fraction (LVEF)

Major adverse cardiac events (MACEs)

Mesenchymal stem cells (MCSs)

Myocardial infarction (MI)

Serious adverse events (SAEs)

Wharton's jelly mesenchymal stem cells (WJ-MSCs)

\section{Declarations}

Ethics approval and consent to participate: We discussed all ethical issues with the Institutional Review Board of Shiraz University of Medical Sciences, which ultimately approved the study protocol (IR.SUMS.REC.1400.409). Informed consent will be obtained once patients are clinically stable and sedatives or strong analgesics do not alter their consciousness.

Consent for publication: Written consent will be obtained from all patients for publication of their data following deidentification. 
Data availability: Data will be made available upon reasonable request following the completion of the study.

Competing interests: M. V. is the regulatory affairs manager in Cell Tech Pharmed. Cell Tech Pharmed Co. will provide us with cGMP grade WJ-MSCs but is not involved in the design or interpretation of the research.

Funding: We have received funding from the Office of the Vice-Chancellor for Research of Shiraz University of Medical Sciences (grant numbers SG-98-5, SG-98-94, and SG-96-86). This study is also supported by the National Institute of Medical Research Development (NIMAD; grant No. 4000140).

Authors' contributions: AA, AM, MM, MV, SAH, JK, AA-A, PI, NA, GP, and MR

were involved in protocol development, manuscript preparation, and critical revision. AA and AM designed the study. MV will provide the project with stem cells. All authors accept responsibility and accountability for the whole project.

Acknowledgments: We thank the Shiraz University of Medical Sciences (Shiraz, Iran) and the Center for Development of Clinical Research of Nemazee Hospital.

\section{References}

1. Organization WH. The Global Burden of Disease: 2004 Update. Geneva: World Health Organization2008.

2. Velagaleti RS, Pencina MJ, Murabito JM, Wang TJ, Parikh NI, D’Agostino RB, et al. Long-term trends in the incidence of heart failure after myocardial infarction. Circulation. 2008;118(20):2057-62.

3. Hellermann JP, Jacobsen SJ, Gersh BJ, Rodeheffer RJ, Reeder GS. Heart failure after myocardial infarction: a review. The American journal of medicine. 2002;113(4):324-30.

4. Roger VL, Go AS, Lloyd-Jones DM, Benjamin EJ, Berry JD, Borden WB, et al. Heart disease and stroke statistics-2012 update: a report from the American Heart Association. Circulation. 2012;125(1):e2e220.

5. Yancy CW, Jessup M, Bozkurt B, Butler J, Casey DE, Jr., Colvin MM, et al. 2016 ACC/AHA/HFSA Focused Update on New Pharmacological Therapy for Heart Failure: An Update of the 2013 ACCF/AHA Guideline for the Management of Heart Failure: A Report of the American College of Cardiology/American Heart Association Task Force on Clinical Practice Guidelines and the Heart Failure Society of America. Circulation. 2016;134(13):e282-93.

6. Juillière Y, Cambou JP, Bataille V, Mulak G, Galinier M, Gibelin P, et al. Heart failure in acute myocardial infarction: a comparison between patients with or without heart failure criteria from the FASTMl registry. Rev Esp Cardiol (Engl Ed). 2012;65(4):326-33. 
7. Lewis EF, Moye LA, Rouleau JL, Sacks FM, Arnold JM, Warnica JW, et al. Predictors of late development of heart failure in stable survivors of myocardial infarction: the CARE study. J Am Coll Cardiol. 2003;42(8):1446-53.

8. Juillière $Y$, Cambou JP, Bataille V, Mulak G, Galinier M, Gibelin P, et al. Heart failure in acute myocardial infarction: a comparison between patients with or without heart failure criteria from the FASTMI registry. Revista Española de Cardiología (English Edition). 2012;65(4):326-33.

9. Braunwald E. Cell-Based Therapy in Cardiac Regeneration: An Overview. Circ Res. 2018;123(2):1327.

10. Taylor DA, Atkins BZ, Hungspreugs P, Jones TR, Reedy MC, Hutcheson KA, et al. Regenerating functional myocardium: improved performance after skeletal myoblast transplantation. Nat Med. 1998;4(8):929-33.

11. Leor J, Patterson M, Quinones MJ, Kedes LH, Kloner RA. Transplantation of fetal myocardial tissue into the infarcted myocardium of rat. A potential method for repair of infarcted myocardium? Circulation. 1996;94(9 Suppl):li332-6.

12. Orlic D, Kajstura J, Chimenti S, Jakoniuk I, Anderson SM, Li B, et al. Bone marrow cells regenerate infarcted myocardium. Nature. 2001;410(6829):701-5.

13. Kocher AA, Schuster MD, Szabolcs MJ, Takuma S, Burkhoff D, Wang J, et al. Neovascularization of ischemic myocardium by human bone-marrow-derived angioblasts prevents cardiomyocyte apoptosis, reduces remodeling and improves cardiac function. Nat Med. 2001;7(4):430-6.

14. Menasché $P$, Hagège $A A$, Scorsin $M$, Pouzet $B$, Desnos $M$, Duboc $D$, et al. Myoblast transplantation for heart failure. Lancet. 2001;357(9252):279-80.

15. Assmus B, Schächinger V, Teupe C, Britten M, Lehmann R, Döbert N, et al. Transplantation of Progenitor Cells and Regeneration Enhancement in Acute Myocardial Infarction (TOPCARE-AMI). Circulation. 2002;106(24):3009-17.

16. Williams A, Hare J. Mesenchymal Stem Cells: Biology, Pathophysiology, Translational Findings, and Therapeutic Implications for Cardiac Disease. Circulation research. 2011;109:923-40.

17. Houtgraaf JH, den Dekker WK, van Dalen BM, Springeling T, de Jong R, van Geuns RJ, et al. First Experience in Humans Using Adipose Tissue-Derived Regenerative Cells in the Treatment of Patients With ST-Segment Elevation Myocardial Infarction. Journal of the American College of Cardiology. 2012;59(5):539-40.

18. Houtgraaf JH, den Dekker WK, van Dalen BM, Springeling T, de Jong R, van Geuns RJ, et al. First experience in humans using adipose tissue-derived regenerative cells in the treatment of patients with STsegment elevation myocardial infarction. J Am Coll Cardiol. 2012;59(5):539-40. 
19. Karantalis V, Schulman I, Balkan W, Hare J. Allogeneic Cell Therapy A New Paradigm in Therapeutics. Circulation research. 2015;116:12-5.

20. Hare JM, Fishman JE, Gerstenblith G, DiFede Velazquez DL, Zambrano JP, Suncion VY, et al. Comparison of allogeneic vs autologous bone marrow-derived mesenchymal stem cells delivered by transendocardial injection in patients with ischemic cardiomyopathy: the POSEIDON randomized trial. JAMA. 2012;308(22):2369-79.

21. Heldman AW, DiFede DL, Fishman JE, Zambrano JP, Trachtenberg BH, Karantalis V, et al. Transendocardial mesenchymal stem cells and mononuclear bone marrow cells for ischemic cardiomyopathy: the TAC-HFT randomized trial. JAMA. 2014;311(1):62-73.

22. Traverse JH, Henry TD, Ellis SG, Pepine CJ, Willerson JT, Zhao DX, et al. Effect of intracoronary delivery of autologous bone marrow mononuclear cells 2 to 3 weeks following acute myocardial infarction on left ventricular function: the LateTIME randomized trial. Jama. 2011;306(19):2110-9.

23. Traverse JH, Henry TD, Pepine CJ, Willerson JT, Zhao DX, Ellis SG, et al. Effect of the use and timing of bone marrow mononuclear cell delivery on left ventricular function after acute myocardial infarction: the TIME randomized trial. Jama. 2012;308(22):2380-9.

24. Fisher SA, Zhang H, Doree C, Mathur A, Martin-Rendon E. Stem cell treatment for acute myocardial infarction. The Cochrane database of systematic reviews. 2015(9):Cd006536.

25. Gao LR, Chen Y, Zhang NK, Yang XL, Liu HL, Wang ZG, et al. Intracoronary infusion of Wharton's jelly-derived mesenchymal stem cells in acute myocardial infarction: double-blind, randomized controlled trial. BMC medicine. 2015;13:162.

26. Jeong H, Yim HW, Park HJ, Cho Y, Hong H, Kim NJ, et al. Mesenchymal Stem Cell Therapy for Ischemic Heart Disease: Systematic Review and Meta-analysis. International journal of stem cells. 2018;11(1):1-12.

27. Sanganalmath S, Bolli R. Cell Therapy for Heart Failure: A Comprehensive Overview of Experimental and Clinical Studies, Current Challenges, and Future Directions. Circulation research. 2013;113:810-34.

\section{Figures}




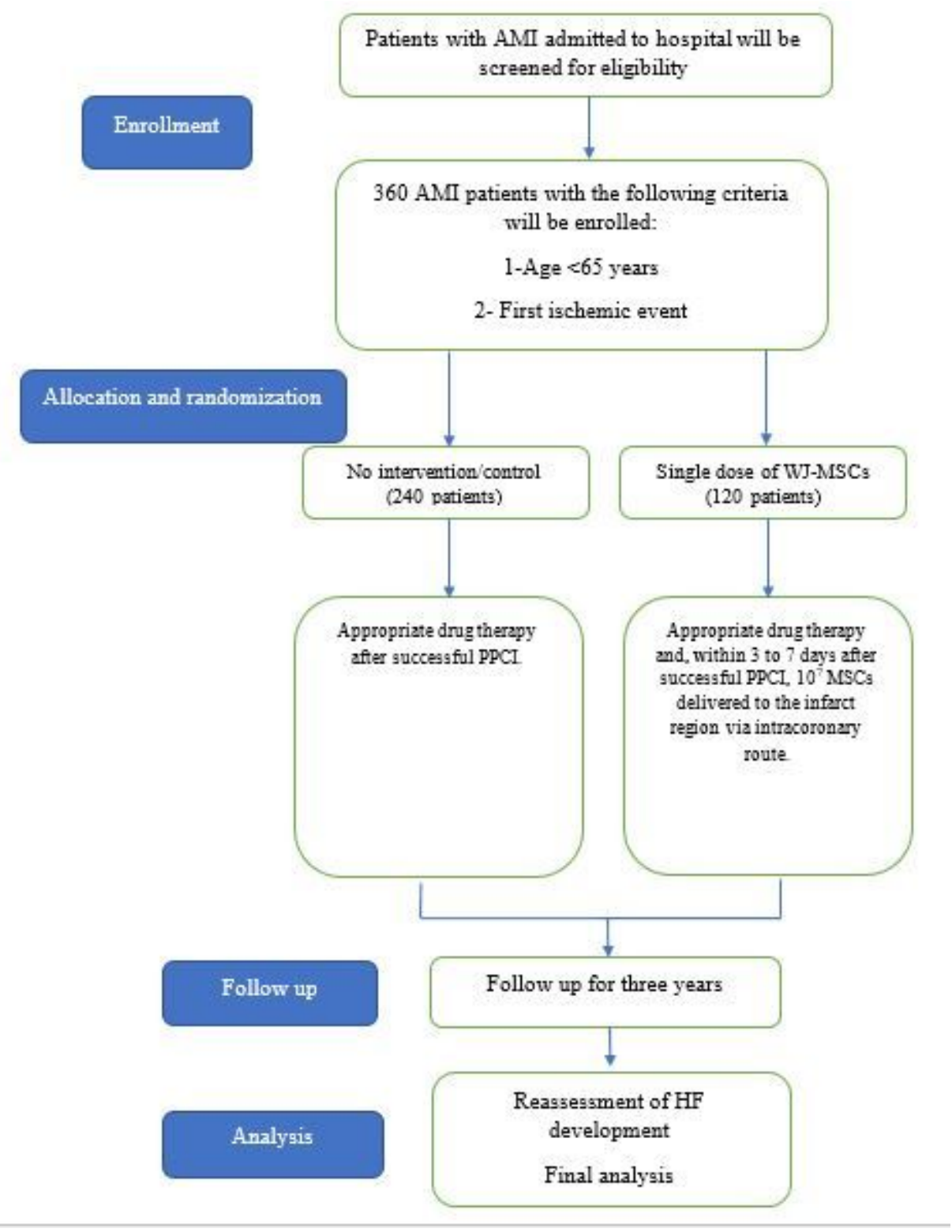

Figure 1

SPIRIT flow diagram of the study.

\section{Supplementary Files}

This is a list of supplementary files associated with this preprint. Click to download.

- SPIRITChecklistFilled.pdf 